\title{
High Resolution Measurements of Isotope Shifts and Hyperfine Structure in Stable and Radioactive Lead Isotopes
}

R. C. Thompson, M. Anselment, K. Bekk, S. Göring, A. Hanser, G. Meisel, H. Rebel, G. Schatz, B. A. Brown Institut für Angewandte Kernphysik 

KERNFORSCHUNGSZENTRUM KARLSRUHE

Institut für Angewandte Kernphysik

KfK 3455

HIGH RESOLUTION MEASUREMENTS OF ISOTOPE SHIFTS AND HYPERFINE STRUCTURE IN STABLE AND RADIOACTIVE LEAD ISOTOPES

R.C. Thompson, M. Anselment, K. Bekk, S. Göring, A. Hanser, G. Meisel, H. Rebel, G. Schatz and B.A. Brown ${ }^{+}$

+ Michigan State University, East Lansing, Michigan 48824, USA Kernforschungszentrum Karlsruhe $\mathrm{GmbH}$, Karlsruhe 
Als Manuskript vervielfältigt

Für diesen Bericht behalten wir uns alle Rechte vor

Kernforschungszentrum Karlsruhe $\mathrm{GmbH}$

ISSN 0303-4003 


\section{ABSTRACT}

We present new measurements of isotopic shifts and hyperfine structure in the lead resonance line for a total of 15 isotopes. The experimental accuracy is of order $4 \mathrm{MHz}$. Using independent measurements of the nuclear parameter $\lambda$ for the stable isotopes we have derived $\lambda$ for all measured isotopes. The derived $\lambda$ values are compared with various theoretical predictions for the lead nuclei. We also give values for the nuclear magnetic dipole and electric quadrupole moments deduced from our measurements.

HOCHAUFLÖSENDE MESSUNGEN DER ISOTOPIEVERSCHIEBUNG UND HYPERFEINSTRUKTUR IN STABILEN UND RADIOAKTIVEN BLEI-ISOTOPEN

Wir berichten über Messungen der Isotopieverschiebung und Hyperfeinstruktur der Blei-Resonanzlinie für insgesamt 15 Isotope. Die experimentelle Genauigkeit liegt in der Größenordnung $4 \mathrm{MHz}$. Mit Hilfe unabhängiger Ergebnisse über die Werte des nuklearen Parameters $\lambda$ bei stabilen Isotopen wurden $\lambda$-Werte für alle gemessenen Isotope bestimmt. Die gefundenen Werte für $\lambda$ wurden mit theoretischen Vorausiagen verschiedener Kernstrukturmodelle verglichen. Zusätzlich wurden aus unseren Messungen Werte für die elektromagnetischen Kernmomente abgeleitet. 


\section{INTRODUCTION}

Optical spectra are a source of information on the properties of the atomic nucleus, and have historically been very important in our understanding of the nucleus. Optical isotope shifts measurements are used to study changes of nuclear size, while the hyperfine splitting of spectral lines is used to determine nuclear moments. One great advantage which optical methods have over others is their great sensitivity. Whereas, for example, scattering experiments typically call for milligram samples of enriched isotopes, optical methods require much smaller quantities, and can thus be extended to radioactive isotopes of which only minute quantities can be prepared. We have used a laser spectroscopic method to study a series of stable and unstable lead isotopes. Lead has been of particular interest in isotope shifts studies since the early days of spectroscopy (e.g. Kopferman 1932) and recent work has included two-photon measurements (Lindgren et al.1982), atomic beam laser spectroscopy (Hüffer 1982) and absorption studies of some radioactive species (Moscatelli et al. 1982). There is a long chain of lead isotopes which can be studied by off-line techniques and this gives information on changes of nuclear mean square charge radii as well as on nuclear moments. Several independent measurements of the charge radii of the stable isotopes are available for calibration of the isotope shifts data. The variation of the charge radii in lead is of particular interest because it enables to test critically theoretical models in the region of a double shell closure $\left({ }^{208} \mathrm{~Pb}\right)$, where calculations are physically very instructive. For comparison with the experimental results one can either adopt a microscopic approach in order to calculate the nuclear densities, or use a macroscopic approach such as the droplet model. (Myers 1977)

In a preceeding paper (Thompson et al. 1982) we presented results of isotope shift and hyperfine structure measurements for 10 stable and radioactive lead isotopes. A preliminary analysis of the isotope shifts in terms of changes of mean 
square charge radii was also given. In this paper we present final results for a total of 15 isotopes (including one isomeric state) and we include a fuller description of the experimental techniques and a detailed analysis of the measurements as well as a discussion of the results in the light of the various nuclear structure models of the lead nuclei.

\section{EXPERIMENTAL SET-UP}

A block diagram of the apparatus is shown in figure 1. The experiment is based on the observation of fluorescence from a well-collimated atomic beam. The fluorescence was induced in the lead resonance line $(283.3 \mathrm{~nm})$ by the frequency-doubled output of a dye laser, whose frequency was scanned across the atomic absorption profile.

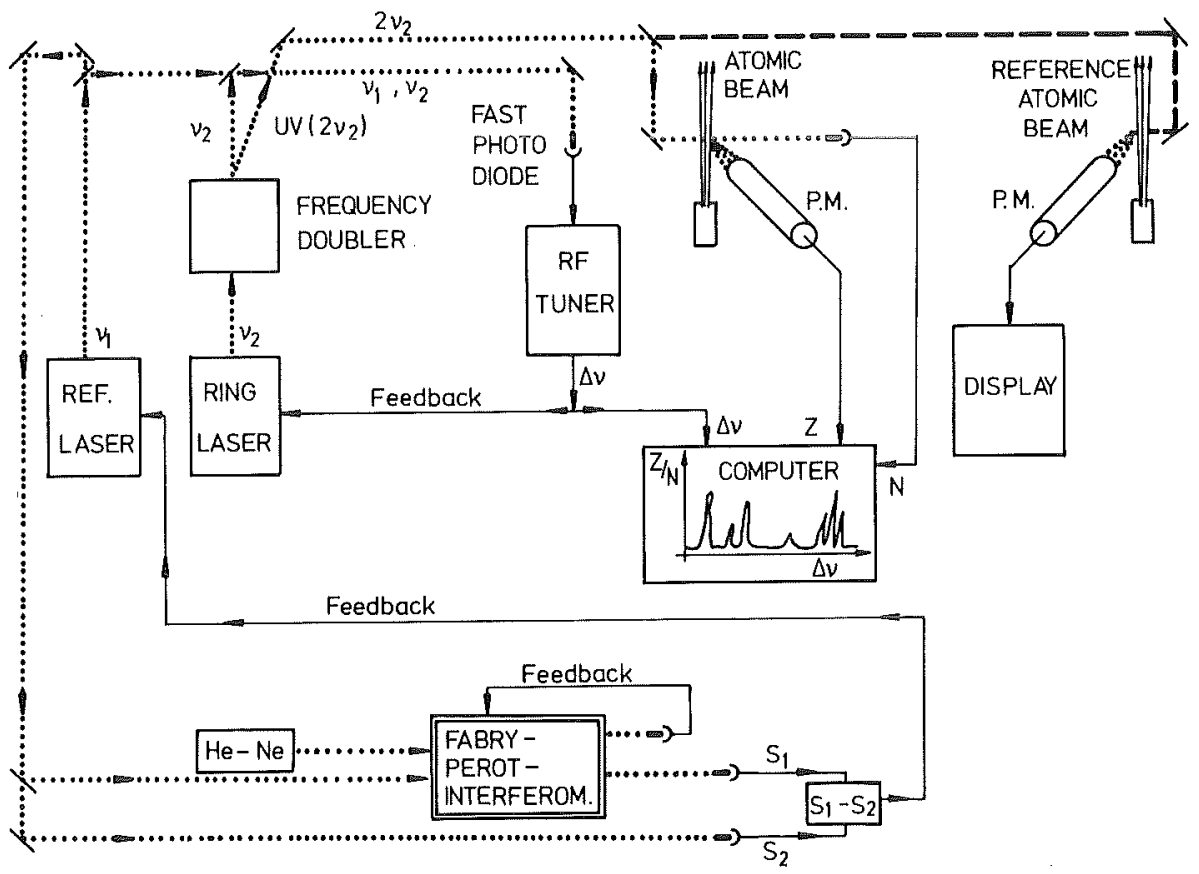

Fig. 1: Schematic view of the experimental set-up used for spectroscopy of $\mathrm{Pb}$. 
We now proceed to describe the major parts of the apparatus in turn.

a. The atomic beam apparatus

The atomic beam apparatus has been described in detail elsewhere (Nowicki et al. 1978). Great care was taken in its construction to reduce the amount of photomultiplier background signal due to scattered laser light and thermal radiation from the atomic beam oven. The windows were placed as far as possible away from the interaction region, and suitable filters were placed in front of the photomultiplier. The fluorescence was monitored at $405.8 \mathrm{~nm}$ to avoid problems with stray laser 1ight; roughly $50 \%$ of the atoms decay by this route. However, since the glass used in the lenses fluoresces at the laser wavelenght, good control of the position of the laser beam was still necessary.

The photomultiplier (EMI model 9558 used in the photon-counting mode) had to be placed well away from the interaction region because of gamma radiation from our samples. With a imaging system of aperture $0.28 \times 4 \pi$ we were able to have the photomultiplier approximately $0.5 \mathrm{~m}$ away. It was well shielded with lead bricks to reduce the background. With this arrangement the $\gamma$-ray background was at worst two or three hundred counts per second, and for most of the samples much less than this. Background from all other sources was also reduced well below this level by an interference filter with a transmission of $40 \%$ at the $405.8 \mathrm{~nm}$ detection wave length. With the large collection solid angle we estimate an overall quantum efficiency of the detection system of about 3 응. It was found necessary to have a liquid nitrogen cooled catcher for the lead beam behind the interaction region. Otherwise a doppler-broadened signal from a "cloud" of lead would completely swamp the signal from a small sample.

b. Generation and irradiation of UV laser light The lead resonance line $\left(6 \mathrm{p}^{2}{ }^{3} \mathrm{p}_{0}-6 \mathrm{p} 7 \mathrm{~s}{ }^{3} \mathrm{P}_{1}^{0}\right)$ is at $283.3 \mathrm{~nm}$ which is in a wavelength range directly inaccessible to $\mathrm{CW}$ tunable lasers. We therefore adopted the frequency-doubling technique: the output of a ring dye laser (Coherent model 699) 
operating at $566.7 \mathrm{~nm}$ was focussed into a non-linear crystal (ADA) which then generated a small amount of light at the second harmonic of the input frequency. This process was optimised by temperatur-tuning the crystal so that the 90-degree phase-matching condition was fulfilled (Boyd and Kleinman 1968). The temperature of the crystal was roughly $-45^{\circ} \mathrm{C}$ and was held constant to better than $0.1^{\circ} \mathrm{C}$. The beam waist inside the crystal was also chosen to optimise the output power (Boyd and Kleinman 1968).

The ring laser was pumped with an argon-ion laser running on the green line $(514.5 \mathrm{~nm})$ at about $7 \mathrm{~W}$. At $566.7 \mathrm{~nm}$ we obtained of order $700 \mathrm{~mW}$ (single mode) with rhodamine 504 dye. From the frequency doubler we then obtained up to $0.2 \mathrm{mw}$ at $283.3 \mathrm{~nm}$, which was then separated from the visible beam by a quartz prism. The UV beam was reflected directly to the atomic beam, all frequency measurement being performed on the visible beam. Usually a glass filter (UG5) was inserted in the UV beam; this further reduced the photomultiplier dark count by blocking all visible light.

From the experimental linewidth we deduce that the atomic transition begins to saturate at about $0.1 \mathrm{~mW}$ UV power at the interaction region. The powers used were therefore at about the optimum, which means that at the peaks of our lines each atom undergoes of order one excitation.

c. Frequency control and measurement

The aim of the experiment is to measure frequency differences between the resonances of the various isotopes, and between the hyperfine components of the odd isotopes. In order to do this we established an optical reference frequency for each experiment, and measured the positions of the various lines relative to this reference frequency. Thus we arrived at difference frequencies between the components.

The reference frequency is generated by a second dye-laser running at $566.7 \mathrm{~nm}$. This laser is stabilised by locking it to the side of a transmission peak of a high finesse $2 \mathrm{GHz}$ cavity, which is temperature-stabilised to reduce long-term drift. For overall stability, the cavity is locked lagain, on 
the side of a transmission peak) to a frequency-stabilised He-Ne laser (Tropel model 200). Medium-terms (5 min) changes of the apparent positions of the atomic lines ( $\sim 3 \mathrm{MHz}$ in the visible) arise from frequency drift of the He-Ne laser, representing approximately its quoted specification.

The ring dye laser is frequency locked to its own reference cavity; its linewidth is of order $1 \mathrm{MHz}$. Its frequency can be changed by application of a voltage to its control electronics. The outputs of the two dye lasers are mixed on a fast photodiode whose output is therefore modulated at the difference frequency $\nu$ of the two lasers. This signal is analysed by an rf spectrum analyser (HP model 8555A) whose central frequency $\nu_{0}$ is controlled by an external voltage generated by an online computer. The spectrum analyser sweeps repeatedly around its central frequency (a range of $20 \mathrm{MHz}$ ) and if $\nu$ lies within this range an output is produced which, by its phase relative to the scan voltage, contains information as to the relative positions of $\nu$ and $\nu_{0}$. Thus, a phase sensitive detector can be used to generate an error signal which is integrated and fed back to the ring laser to move $v$ towards $v_{0}$. In this way the difference frequency was locked to the preset frequency $v_{0}$. The laser could now be scanned by simultaneously applying ramp voltages under computer control to both the spectrum analyser (to change $\nu_{0}$ ) and the ring laser (to change $v$ ). Any mismatch was taken up by the lock circuit described.

The rf spectrum analyser drive voltage was calibrated for each experimental run by recording the positions of the output frequencies of a $100 \mathrm{MHz}$ comb generator (which was stable to at least one part in $10^{5}$, and interpolating the frequency scale.

For the measurements on $199,202 \mathrm{~m}, 209,212 \mathrm{~Pb}$, of which we could produce only very small samples, we adopted a different frequency control method in order to obtain rough ( $\pm 20 \mathrm{MHz})$ values for the positions of the components. This was done since the atomic beam from these samples did not last long enough for the application of the more accurate heterodyne method outlined above. We used the ring laser alone in its internal 
scan mode to sweep it over the whole range interest including usually one or several of the stable isotopes for calibration. A multi-channel analyser (in multi-scaling mode) was scanned synchronously with the laser and recorded the photon count as a function of laser frequency. Usually five scans leach lasting $3.5 \mathrm{~s})$ were accumulated and recorded on magnetic tape and then the cycle was repeated.

The advantage of this method was that new peaks could be located even if we did not know exactly where to look for them. With the heterodyne method it took time to find the peaks initially; with a small sample one could lose a lot of valuable time this way. Therefore this method was also applied to new isotopes before the heterodyne measurements were performed.

Since the internal laser scan was slightly non-linear, the spectra were all linearised by recording the transmission of a high finesse cavity (of free spectral range roughly $300 \mathrm{MHz}$ ) under the same conditions and using its transmission maxima as equally spaced frequency markers of known distance.

\section{d. Sample preparation}

The samples were all prepared with the aid of an electromagnetic mass separator. This ensured that almost only the desired isotopes were present in the sample and also allowed us to add controlled amounts of the stable isotopes. ${ }^{210} \mathrm{~Pb}$ is readily available, as it is a long-lived natural decay product (RaD); $212 \mathrm{~Pb}$ (ThB) was collected from the decay of ${ }^{232} \mathrm{Th}$; and ${ }^{205} \mathrm{~Pb}$ was produced in the Karlsruhe Research Reactor FR2 by neutron irradiation. All the other radioactive isotopes were made at the Karlsruhe Isochronous Cyclotron. 201, 202m, $203 \mathrm{~Pb}$ were produced by irradiating $T 1$ targets with deuterons of favourable energies ( 38 or $48 \mathrm{MeV}$ ); $198,200,202 \mathrm{~Pb}$ were produced from $\mathrm{Hg}$ targets by alpha-irradiation at $104 \mathrm{MeV}$ and ${ }^{209} \mathrm{~Pb}$ by ${ }^{208} \mathrm{~Pb}(\mathrm{~d}, \mathrm{p})$ reactions.

From the irradiated $\mathrm{TI}$ and $\mathrm{Hg}$ targets the lead activity was first separated by a chemical procedure (TI) or distillation $(\mathrm{Hg})$ and then underwent mass separation. The ions emerging 
from the separator were implanted directly in the atomic beam oven (made of tantalum). This oven could be inserted into the atomic beam apparatus under vacuum, thus ensuring speedy measurement of a sample after preparation. The samples contained typically of the order $1 \mathrm{ng}$ the isotope under study and matching amounts of the relevant stable reference isotopes; it was possible to make measurements. on samples of the order of

$50 \mathrm{pg}$. The smallest samples used so far were those of $209,212 \mathrm{~Pb}$ (approximately $50 \mathrm{pg}$ ); in these cases the radioactive atomic beams lasted for about on ly 30 seconds allowing for a total of eight useful scans in the internal sweep mode.

\section{e. Experimental procedure}

The isotope shifts between neighbouring even isotopes are of order $2 \mathrm{GHz}$ (all frequency differences quoted are for the atomic (UV) wavelength, unless otherwise stated). Since the photodiode worked only up to $~ 3 \mathrm{GHz}$ frequency difference we could scan up to $6 \mathrm{GHz}$ in the UV. This is not enough to cover the spectrum of all the isotopes. We therefore measured the positions of the stable isotopes and used these positions as reference points, measuring the other isotopes relative to the nearest stable one.

When the heterodyne frequency control was used we recorded many spectra from each sample. This was because of the He- Ne reference frequency drift mentioned above. If one measures the positions of two components many times (in the sequence $A, B, B, A \ldots)$ one can reduce the uncertainty due to the reference frequency changing slowly. Each component was recorded with data points spaced by typically $8 \mathrm{MHz}$ at a rate of about one point per second; the line profile was recorded well out into the wing so that a least squares fit could be performed on it; a jump was then made directly to the next component in order to reduce the time needed for each spectrum. The experimental linewidths were of order $40 \mathrm{MHz}$ (the natural linewidth is $28 \mathrm{MHz}$ ). Typical peak count rates for the small samples were 5-10 $\mathrm{kHz}$. We had very few problems with overlapping peaks as the isotope shifts and hyperfine structure are so large. A typical spectrum from a ${ }^{201} \mathrm{~Pb}$ sample $(1.2 \mathrm{ng})$ is shown in fig. 2 . 


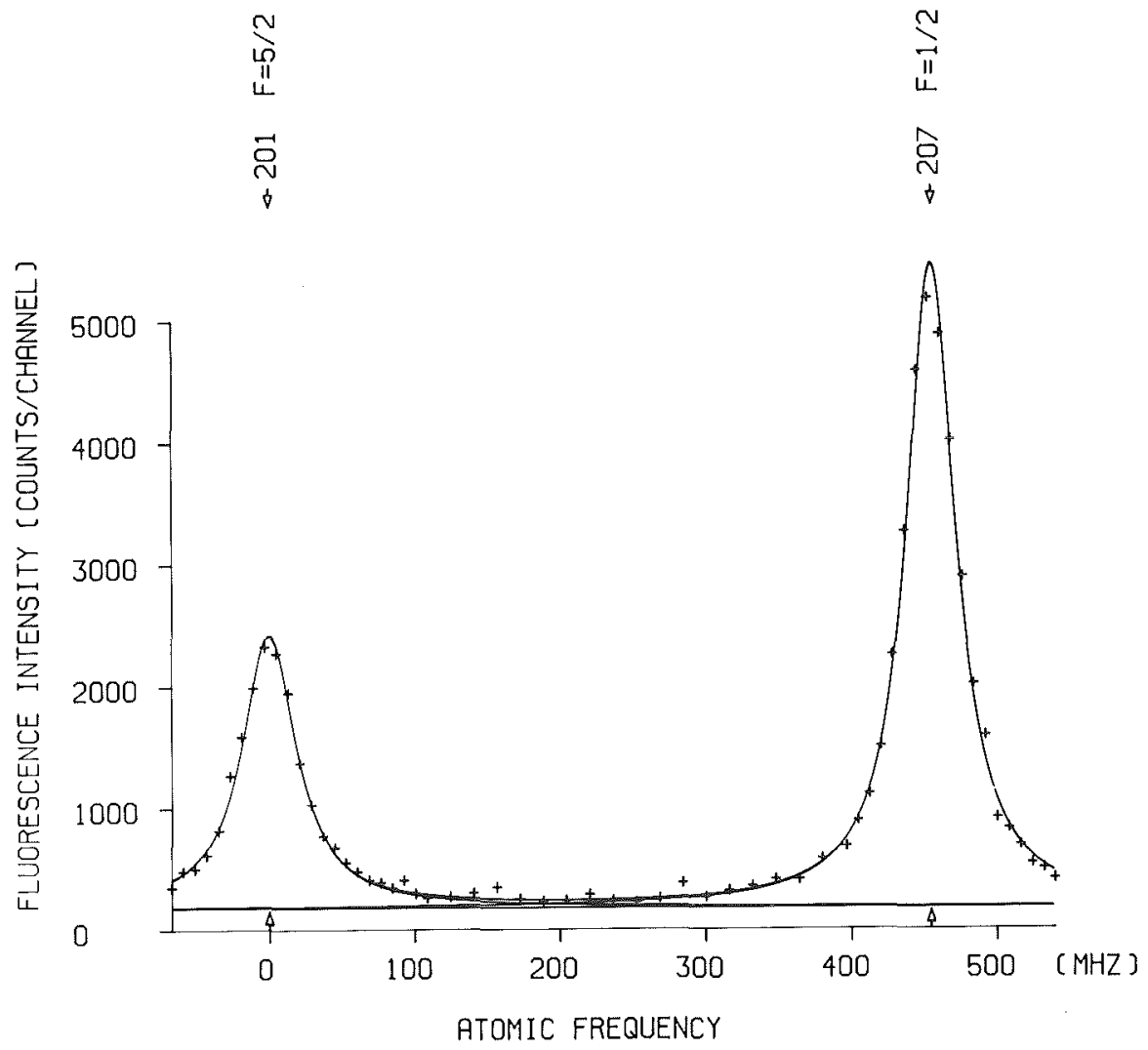

Fig. 2 Laser induced fluorescence spectrum from a sample

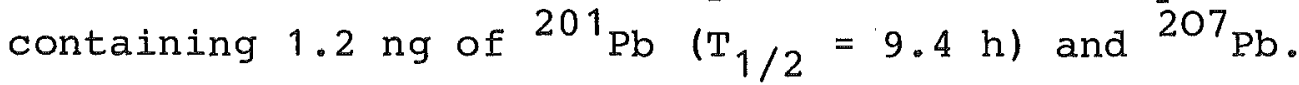

\section{MEASUREMENTS}

As already discussed, we measured first the relative positions of all the stable isotopes $(204,206,207,208 \mathrm{~Pb})$ in order to establish a set of reference points relative to which we could measure the other isotopes. The odd isotopes generally had three components (apart from ${ }^{207} \mathrm{~Pb}$ ) with a spread of order $12 \mathrm{GHz}$. Measurement of all three components therefore generally involved resetting the frequency band of the spectrum analyser and often relocking of the reference laser on a different transmission peak of the $2 \mathrm{GHz}$ cavity.

The results of all measurements are presented in tables 1 and 2. Table 1 shows the isotope shifts (all given relative to ${ }^{208} \mathrm{~Pb}$ ) and their errors. Table 2 gives the $\mathrm{A}$ and $\mathrm{B}$ factors as derived from the hyperfine structure of the odd isotopes. 
For some isotopes that were measured with the more accurate beat method the errors are larger than for others since there are up to three links in the frequency chain from some components to the ${ }^{208} \mathrm{~Pb}$ line. The isomer $202 \mathrm{~m} \mathrm{~Pb}$ as well as 199,209 , ${ }^{212} \mathrm{~Pb}$ were all measured only with the less accurate frequencymarker method using the internal laser sweep, which is the reason for the relatively large errors of the latter ones. The isomeric shift between ${ }^{202} \mathrm{~Pb}$ and $202 \mathrm{~m} \mathrm{~Pb}$ has been found to be $37.7(4.0) \mathrm{MHz}$. A spectrum for the isomer ${ }^{202} \mathrm{~Pb}$ obtained by the sweep method (see sect.2c) is shown in Fig.3; the sample here was of the order of $700 \mathrm{pg}$.

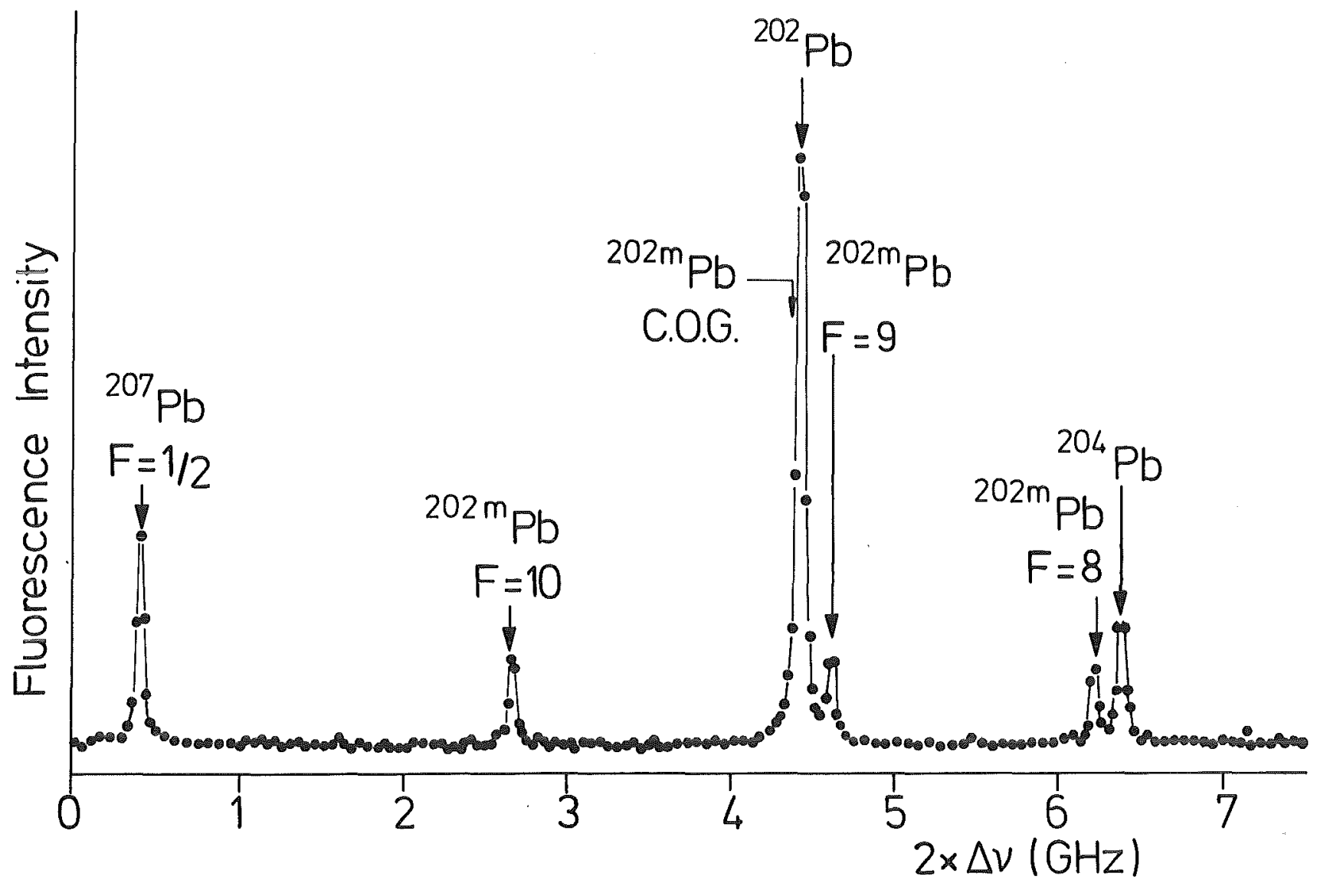

Fig. 3 Laser spectroscopy of the ${ }^{202 \mathrm{~m}} \mathrm{~Pb}-{ }^{202} \mathrm{~Pb}$ isomeric shift. 


\begin{tabular}{lll} 
Isotope & $\begin{array}{c}\text { Isotope shift } \\
{[\mathrm{MHz}]}\end{array}$ & $\begin{array}{c}\text { Nuclear parameter }(\lambda) \\
{\left[\mathrm{fm}^{2}\right]}\end{array}$ \\
\hline 198 & $-9848.4(5.0)$ & $-.491(1)$ \\
199 & $-9748 .(9.0)$ & $-.4813(51)$ \\
200 & $-8094.1(3.5)$ & $-.4018(67)$ \\
201 & $-7727.6(5.0)$ & $-.3807(38)$ \\
202 & $-6193.7(3.5)$ & $-.3067(41)$ \\
$202 \mathrm{~m}$ & $-6230.4(5.0)$ & $-.3084(39)$ \\
203 & $-5749.0(5.0)$ & $-.2821(33)$ \\
204 & $-4212.0(2.5)$ & $-.2080(23)$ \\
205 & $-3712.6(3.0)$ & $-.1810(37)$ \\
206 & $-2226.6(2.5)$ & $-.1094(12)$ \\
207 & $-1390.0(2.5)$ & $-.0672(23)$ \\
208 & 0.0 & .0 \\
209 & $+1767(90)$ & $+.0842(48)$ \\
210 & $+3973.7(3.5)$ & $+.188(13)$ \\
212 & $+7815(30)$ & $+.371(25)$
\end{tabular}

Table 1: Measured isotope shifts (relative to ${ }^{208} \mathrm{~Pb}$ ) and derived values of $\lambda$, the nuclear parameter, for the lead resonance line $(283.3 \mathrm{~nm})$. The isotope shifts were calibrated using the data of Fricke and Rychel (1981) for the nuclear parameter of the stable isotopes.

Our results are generally consistent with the classical spectroscopy results of Moscatelli et al. (1981, 1982), but our error limits are an order of magnitude smaller and we have been able to study a larger number of isotopes.

\section{DERIVATION OF NUCLEAR QUANTITIES}

a. Changes of mean square charge radius

As is well known, the isotope shifts between two isotopes in a spectral line can be expressed as follows (e.g. Kuhn 1969): 


$$
\delta \nu_{A A^{\prime}}=\mathbb{M} \frac{A-A^{\prime}}{A A^{\prime}}+F \lambda_{A A^{\prime}}
$$

The first term (the mass shift) depends only on the masses of the two isotopes; the second term (the field shift) depends on the nuclear parameter $\lambda$. This can be expressed as a power series of mean charge radii differences (Seltzer 1969):

$$
\lambda=\delta\left\langle r^{2}\right\rangle+\left(C_{2} / C_{1}\right) \delta\left\langle r^{4}\right\rangle+\left(C_{3} / C_{1}\right) \delta\left\langle r^{6}\right\rangle \ldots
$$

Forlight elements the higher order terms can be neglected, but for lead they are significant. Using standard theoretical models for the charge distribution (without deformations), one can obtain values for the ratios $\delta\left\langle r^{4}\right\rangle / \delta\left\langle r^{2}\right\rangle$ and $\delta\left\langle r^{6}\right\rangle / \delta\left\langle r^{2}\right\rangle$ and combine these with the coefficients tabulated by seltzer to obtain:

$$
\lambda=\delta\left\langle r^{2}\right\rangle(1-0.084+0.014)=0.93 \delta\left\langle r^{2}\right\rangle
$$

which we estimate to be accurate to at least $2 \%$. However, if there are deformations present, this relation will no longer hold as the values of the higher order moments for a given $\delta\left\langle r^{2}\right\rangle$ will be different.

In order to extract $\lambda$ from the experimental results we need independent knowledge of the electronic factor $F$ and the mass shift $M$. It is obtained by combining optical isotope shifts data with independently determined values of $\lambda$ or $\delta\left\langle r^{2}\right\rangle$ for at least two isotope pairs $\left(A, A^{\prime}\right)$. For this purpose data from electronic $x$-ray, and combined electron scattering and muonic $\mathrm{X}$-ray measurements are available.

Eq. 4.1 can be written as

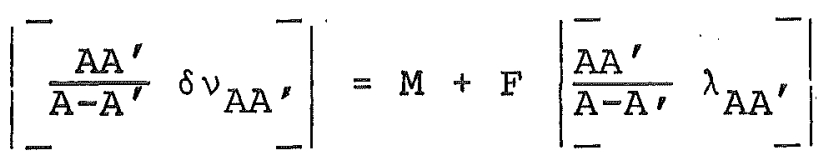

so that a plot of the two items in square brackets against each other for different isotope pairs yields a straight line of intercept $M$ and slope $F$. This so-called King plot contains as many points as there are isotope pairs for which $\lambda$ has been 
determined (usually all the stable isotopes); the point is that the derived values of $F$ and $M$ can be used to calculate $\lambda$ for every isotope for which an optical measurement has been made. Thus the calibration carried out using a few isotopes can then be applied to all isotopes. The result is values of $\lambda$ for the radioactive isotopes and improved values for the stable isotopes as well.

We have performed this analysis with three different sets of data.

(i) The muonic X-ray data of Kesseler et al. (1975) are notable in that they show no odd-even staggering between isotopes 206, 207 and 208, while our data show a pronounced staggering. Consequently, the derived value of $M$ is rather large: equivalent to $-700(1300)$ times the normal mass shift (nms), which would represent the value of $M$ in the absence of many-body effects. It is highly unlikely that the total mass shift is so much different from the nms: the specific mass shift (sms), which represents the many-body contribution to the total mass shift, is usually of the same order of or less than the nms. In our case, the nms is only $-26.9 \mathrm{MHz}$ between ${ }^{206} \mathrm{~Pb}$ and ${ }^{208} \mathrm{~Pb}$, compared to the total isotope shift of -2226.6 . $\mathrm{MHz}$.

(ii) The data of Borchert et al. (1982), on the other hand, obtained from electronic X-rays, show a pronounced staggering, and the derived value for $M$ is 22.7(3.4) times the nms. This, too, seems unreasonable; King plots of our own data against other optical data (Brix et al. 1952, Lindgren 1981, Hüffer 1982) show evidence for an sms of order \pm 5 times the nms.

(iii) Fricke and Rychel (1981) have determined $\lambda$ in a combined analysis of electron scattering and muonic $x$-ray transitions, and if we use these data we obtain $M=-7.1(13.1)$ times the nms. This is fully consistent with the other optical data and we have used it for our calibration. For $F$ we then obtain $22.1(3.3) \mathrm{GHz}^{\circ} \mathrm{fm}^{-2}$. 


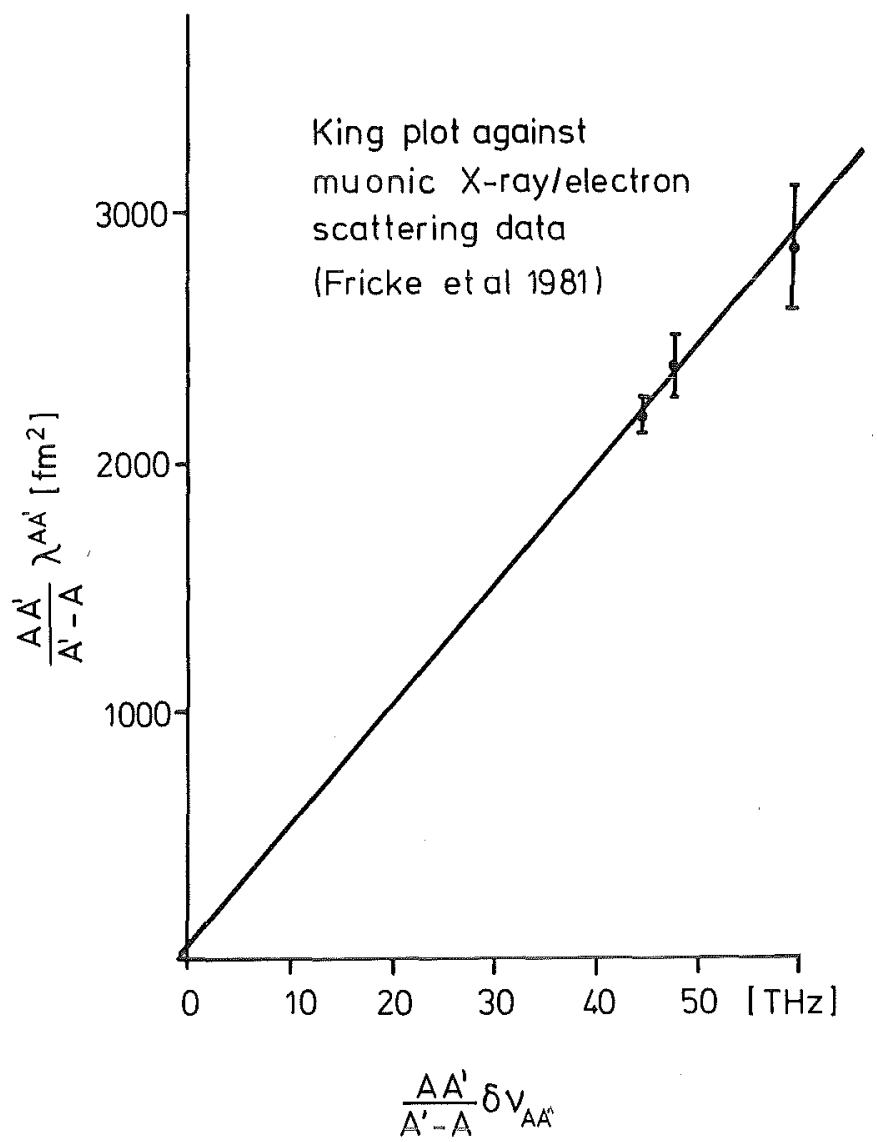

Fig. 4: King plot of the $6 s^{2} 6 p^{2}{ }^{3} P_{0}-6 s^{2} 6 p 7 s{ }^{3} P_{1}^{0}$ resonance transition in $\mathrm{Pb}$ against the nuclear parameter $\lambda_{\mathrm{AA}}$, as derived from a combined analysis of electron scattering and muomic X-ray data (Fricke and Rychel 1981).

Although the errors in $M$ and $F$ seem large, they are highly correlated, and the errors given in Table 1 for the values of $\lambda$ have been determined in a fully consistent manner from the uncertainty in the position of the calibration line at each point. The quoted errors therefore contain all sources of error and are also correlated with each other. There is no external constraint on the data requiring the mass shift to be within certain limits, as there was in our previous paper (Thompson et al. 1982). The difference in $\lambda$ for the isomer pair $202,202 \mathrm{~m}_{\mathrm{Pb}}$ is determined to be $0.0017(4) \mathrm{fm}^{2}$. 
The statistical errors in $\lambda$ arising from measurement uncertainty have been left out of Table 1 as they are all less than $\pm .0003 \mathrm{fm}^{2}$. This shows also that the uncertainty in the calibration is due entirely to the uncertainty in the independent measurements of $\lambda$.

One parameter apart from the value of $\lambda$ itself which is of interest for the odd isotopes is the staggering parameter $\gamma$. This is defined for an odd isotopic A by

$$
\gamma=2\left(\left\langle r^{2}\right\rangle_{A}-\left\langle r^{2}\right\rangle_{A-1}\right) /\left(\left\langle r^{2}\right\rangle_{A+1}-\left\langle r^{2}\right\rangle_{A-1}\right)
$$

and is therefore 1 if there is no staggering and zero if the odd isotope has a charge radius equal to that of the next lowest isotope. Since this quantity consists of a ratio of charge radii differences, it can be deduced without any knowledge of $F$, though in order to obtain it from our data we must assume that $\lambda$ and $\delta\left\langle r^{2}\right\rangle$ are related by a constant factor, which will introduce some uncertainty. Some assumption must also be made about $M$; however, we do not agree with Moscatelli et al. (1982), who have set $M=0$, as we believe that there is strong optical evidence for the sms being significantly greater than the nms (see above). Rather, we impose a limit on the sms of \pm 10 times the nms, i:e.

$$
M=(1 \pm 10) \times \mathrm{nms}
$$

and with these constraints we have been able to evaluate the staggering parameter for the odd isotopes (see Table 2). Again the measurement error is negligible compared to the systematic errors. For comparison, the value of the staggering parameter for ${ }^{207} \mathrm{~Pb}$ is $1.04(17)$ as derived from the data of Kesseler (1975) and $0.66(8)$ from the data of Borchert et al. (1982). The data of Fricke and Rychel (1981) yields $0.80(10)$.

b. Nuclear moments and a new result for the ground state spin of ${ }^{199} \mathrm{~Pb}$

In Table 2 we give the $A$ and $B$ factors as derived from the observed spectra of the odd isotopes and ${ }^{202} \mathrm{~Pb}$. Using standard formulae (e.g. Kuhn 1969) we can relate these to the moments of the 
nucleus. For the magnetic dipole moments we have taken the value of $+0.5783 \mathrm{n} . \mathrm{m}$. for ${ }^{207} \mathrm{~Pb}$ as given by Fuller (1976), and have calculated those for the other isotopes using the known spins and measured A factors. This ignores any hyperfine anomaly which may be present. Since the only stable odd isotope, ${ }^{207} \mathrm{~Pb}$, has a spin of $1 / 2$, there are no independent measurements of nuclear quadrupole moments in lead. However, using calculations by Moscatelli (1980), we are able to convert the measured B factors into nuclear quadrupole moments. It is important to note that the factor relating the two is known only to a precision of $\pm 45 \%$; the quoted values are therefore subject to an additional systematic scaling uncertainty of that amount.

\begin{tabular}{lccccc} 
Isotop & $\begin{array}{c}\text { A Factor } \\
{[\mathrm{MHz}]}\end{array}$ & $\begin{array}{c}\text { B Factor } \\
{[\mathrm{MHz}]}\end{array}$ & $\begin{array}{c}\text { Magnetic } \\
\text { Moment } \\
{[\mathrm{n.m.}]}\end{array}$ & $\begin{array}{c}\text { Quadrupole- } \\
\text { Moment } \\
{[\mathrm{e} . \mathrm{b} .]}\end{array}$ & $\begin{array}{c}\text { Staggering } \\
\text { Parameter }\end{array}$ \\
\hline & & & & & \\
199 & $-5322(6)$ & $-9(10)$ & $-1.048(21)$ & $+0.05(5)$ & $0.10(16)$ \\
201 & $+2007.5(2.0)$ & $+1(10)$ & $+0.659(13)$ & $-0.01(7)$ & $0.38(10)$ \\
$202 \mathrm{~m}$ & $-187.9(1.0)$ & $-67(15)$ & $-0.222(4)$ & $+0.36(8)$ & - \\
203 & $+2040.3(2.0)$ & $-11(10)$ & $+0.670(13)$ & $+0.06(5)$ & $0.44(8)$ \\
205 & $+2115.7(1.5)$ & $-26(6)$ & $+0.695(14)$ & $+0.14(3)$ & $0.50(7)$ \\
207 & $+8807.2(3.0)$ & - & +0.5783 & - & $0.75(3)$ \\
209 & $-2433(3)$ & $+31(19)$ & $-1.438(29)$ & $-0.16(10)$ & $0.89(1)$
\end{tabular}

Table 2: Measured values for the $A$ and $B$ factors of the odd isotopes and of the isomer ${ }^{202 \mathrm{~m}} \mathrm{~Pb}$. The derived nuclear moments are also given. The magnetic moments are calculated assuming a value for $\mu_{I}\left({ }^{207} \mathrm{~Pb}\right)$ of to.5783 n.m. as given by Fuller (1976); The $\mu_{I}$ overall error is estimated to be $2 \%$ due to neglect of a possible hfs anomaly and to account for the $\mu_{I}\left({ }^{207} \mathrm{~Pb}\right)$ discrepancy between the NMR and the optical pumping results (Fuller 1976). The electric quadrupole moments are evaluated using the theoretical electronic factor given by Moscatelli (1980). 
The value obtained for the magnetic moment of ${ }^{209} \mathrm{~Pb}$ is in very good agreement with the value for the $99 / 2$ neutron deduced indirectly from the $7^{-}$state in $210_{\mathrm{Bi}}$ (Baba et al. 1972). The deviation of the experimental value from the single-particle (Schmidt) value $\left(-1.91 \mathrm{n} . \mathrm{m}_{\text {) }}\right.$ can be fully explained by core polarisation effects (Arima and Huang-Lin 1972).

A more detailed comment is necessary on our results on ${ }^{199} \mathrm{~Pb}$. From radioactive decay studies a ground state spin of $5 / 2^{-}$has been adopted (Lederer and Shirley 1979). In addition a $3 / 2^{-}$level has been proposed at $19.58 \mathrm{keV}$ excitation energy (Richel et al. 1978 ). Analysis of the measured hyperfine splittings and isotope shift assuming a ground state spin of $5 / 2^{-}$would result in a negative magnetic moment (incompatible with positive Schmidt value of an $5 / 2$ single particle level) and a very large quadrupole moment $(Q=+3.7 \mathrm{eb})$. The corresponding deformation should induce an isotopic shift considerably larger than the observed value. In contrast, analysing the experimental data by assuming a ground state spin of $3 / 2^{-}$leads to a nearly vanishing $B$ factor and small deformation as expected for this region. In addition the sign of the magnetic moment is then in agreement with the expectation from the Schmidt diagram of a $p 3 / 2$ neutron. We therefore conclude that the ground state spin of ${ }^{199} \mathrm{~Pb}$ is $3 / 2^{-}$. This result is not surprising from point of view of systematics of the quasiparticle energies of the $f 5 / 2$ and $p 3 / 2$ levels, which almost degenerate for $\mathrm{A}=199$.

\section{DISCUSSION}

In zeroth order, the addition or removal of neutrons from the ${ }^{208} \mathrm{~Pb}$ doubly closed shell nucleus, of course, gives no isotopic difference in ms charge radii since neutrons have no charge. Actually, the neutrons themselves contribute a little due to their nonzero charge distribution and due to a relativistic spin-orbit correction (Bertozi et al. 1972). These contributions, however, are negligible compared to other effects. The most important mechanism leading to isotopic charge radius differences is the rearrangement of the protons which occurs with the change of the number of neutrons. In first order the 
valence neutrons polarize the core and, in particular, they admix $1 \mathrm{p} 1 \mathrm{~h} \mathrm{O}^{+}$intermediate core states to the expectation value of the one-body monopole operator under consideration (Speth et al. 1974).

Perhaps the most realistic and simplest global model which attempts to account for the proton rearrangement is the droplet model (Myers 1977, 1982), a refinement of the classical liquid drop model. It is based on macroscopic features and effects determining binding energies and sizes of nuclei. The overall trend predicted for $\delta\left\langle r^{2}\right\rangle_{c h}$ by the droplet model is in remarkably good agreement with experiment (see Fig. 5 and Table 3). However, there is of course no fine structure or shell structure present, and we must turn to more refined models to understand such

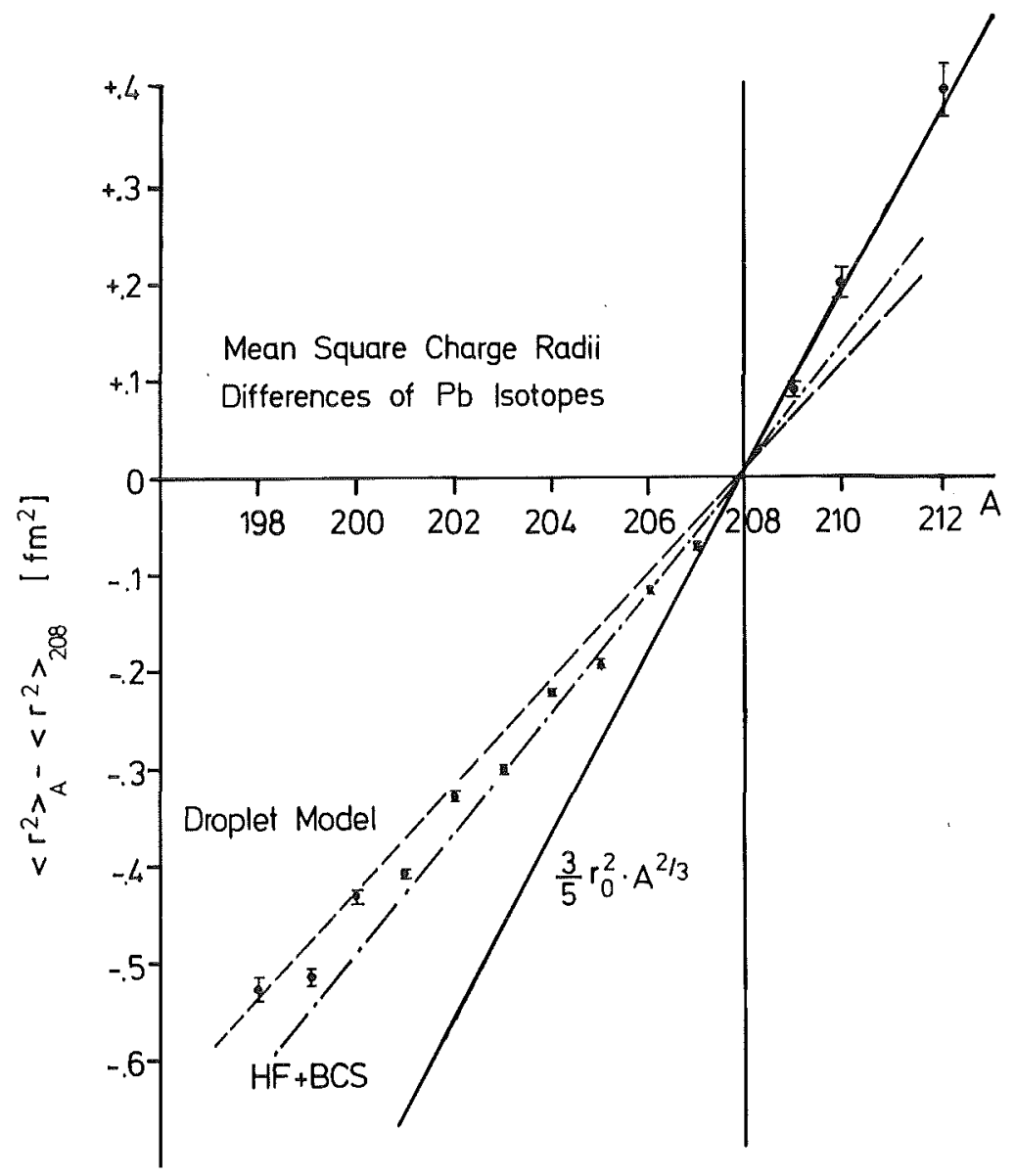

Fig. 5 Mean square charge radii of $\mathrm{Pb}$ isotopes as compared with various theoretical predictions. 
details. It is interesting to note that the droplet model requires a neutron skin in ${ }^{208} \mathrm{~Pb}$, in the sense that the rms radius of the neutron distribution is about $0.2 \mathrm{fm}$ larger than that of the proton distribution.

Speth et al. (1974) have calculated the monopole core polarization using a density-dependent $\delta$-interaction with parameters chosen to reproduce properties of the particle hole states in $208 \mathrm{~Pb}$. The radius variation obtained for addition of one valence neutron is given in Table 4 .

\begin{tabular}{|c|c|c|c|c|c|c|}
\hline \multirow[b]{2}{*}{ Nucleus } & \multicolumn{6}{|c|}{$\delta\left\langle r^{2}\right\rangle_{\mathrm{Ch}}\left[\mathrm{fm}^{2}\right]$} \\
\hline & Exp & $\begin{array}{l}\text { Droplet } \\
\text { Model }\end{array}$ & SIII & SIII $+\mathrm{E} 2$ & Emp.Sh & Emp.Sh+E2 \\
\hline $212_{\mathrm{Pb}}$ & & 0.21 & 0.276 & & & \\
\hline $211_{\mathrm{Pb}}$ & & 0.16 & 0.207 & & & \\
\hline $210_{\mathrm{Pb}}$ & $0.202(14)$ & 0.11 & 0.138 & 0.145 & & \\
\hline $209 \mathrm{~Pb}$ & & 0.05 & 0.060 & & & \\
\hline${ }^{208} \mathrm{~Pb}$ & 0 & 0 & 0 & 0 & & \\
\hline $207 \mathrm{~Pb}$ & $-0.072(2)$ & -0.05 & -0.063 & -0.058 & -0.076 & -0.072 \\
\hline $206_{\mathrm{Pb}}$ & $-0.118(1)$ & -0.11 & -0.125 & -0.114 & -0.152 & -0.139 \\
\hline $205_{\mathrm{Pb}}^{10}$ & $-0-195(4)$ & -0.16 & -0.187 & -0.175 & -0.228 & -0.214 \\
\hline${ }^{204} \mathrm{~Pb}$ & $-0.224(3)$ & -0.21 & -0.249 & -0.229 & -0.304 & -0.281 \\
\hline $203 \mathrm{~Pb}$ & $-0.303(4)$ & -0.27 & -0.310 & & & \\
\hline $202_{\mathrm{Pb}}$ & $-0.332(5)$ & -0.32 & -0.370 & -0.342 & & \\
\hline $201_{\mathrm{Pb}}$ & $-0.410(4)$ & -0.37 & -0.430 & & & \\
\hline $200 \mathrm{~Pb}$ & $-0.432(8)$ & -0.42 & -0.489 & -0.458 & & \\
\hline $199 \mathrm{~Pb}$ & & -0.48 & -0.548 & & & \\
\hline${ }^{198} \mathrm{~Pb}$ & -0.528 & -0.58 & -0.606 & & & \\
\hline
\end{tabular}

Table 3: Comparison of experimental and calculated variations of the nuclear ms charge radii of $\mathrm{Pb}$ nuclei. The values (SIII) for the even nuclei are based on eq. (5.1), the values of the odd nuclei except for ${ }^{209} \mathrm{~Pb}$ (shift for a 2 g9/2 valence neutron) and for ${ }^{207} \mathrm{~Pb}$ (shift for a $3 \mathrm{p} 1 / 2$ valence hole) are obtained from the average of the neighboring even nuclei. +E2: with contributions from 2nd order corrections. Emp.Sh: Using an empirical first order shift of $-0.076 \mathrm{fm}^{2}$. 
Our own calculation used the skyrme interactions III and VI introduced by Beiner et al. (1975). The results are compared with the calculations of Speth et al. (1974, 1981) in Table 4. The various calculations differ very little except for the $1 \mathrm{~h} 9 / 2$ hole neutron state for which speth et al. obtain a very small shift relative to that for the other orbits. We do not understand the reason for this difference. The Skyrme interaction parameters were obtained from fits to the binding energies and radii for many closed-shell nuclei, and hence, like the droplet model, this represents a global parametrization. The difference between the Skyrme III and VI interactions can be related to the effective masses in nuclear matter which are 0.76 and 0.95 respectively. Although an effective mass of 0.76 is close to that obtained from the G-matrix derived from a realistic nucleon-nucleon interaction, the single-particle energy level spacings in ${ }^{208} \mathrm{~Pb}$ are in much better agreement with an effective mass of near unity (Beiner et al. 1975). We see from Table 4 that the orbital

\begin{tabular}{|c|c|c|c|c|}
\hline \multirow[b]{2}{*}{$\mathrm{nlj}$} & \multicolumn{2}{|c|}{$\delta\left\langle r^{2}\right\rangle_{p}\left[f m^{2}\right]$} & \multicolumn{2}{|c|}{$\delta\left\langle r^{2}\right\rangle_{\mathrm{ch}}\left[\mathrm{fm}^{2}\right]$} \\
\hline & Speth & SIII & SIII & SVI \\
\hline $3 d 3 / 2$ & 0.045 & 0.062 & 0.066 & 0.065 \\
\hline $2 g 7 / 2$ & 0.081 & 0.076 & 0.081 & 0.079 \\
\hline $4 s 1 / 2$ & 0.040 & 0.058 & 0.058 & 0.057 \\
\hline $3 d 5 / 2$ & 0.045 & 0.062 & 0.060 & 0.058 \\
\hline $1 \mathrm{j} 15 / 2$ & & 0.063 & 0.056 & 0.054 \\
\hline $1 \mathrm{i} 11 / 2$ & 0.086 & 0.091 & 0.098 & 0.094 \\
\hline $2 \mathrm{~g} 9 / 2$ & 0.075 & 0.064 & 0.060 & 0.059 \\
\hline$\left(3 \mathrm{p} 1 / 2^{-1}\right.$ & -0.061 & -0.061 & -0.063 & -0.065 \\
\hline$(2 \pm 5 / 2)^{-1}$ & -0.053 & -0.968 & -0.072 & -0.074 \\
\hline$(3 \mathrm{p} 3 / 2)^{-1}$ & -0.057 & -0.057 & -0.056 & -0.058 \\
\hline$(1 i 13 / 2)^{-1}$ & -0.066 & $-0-053$ & -0.047 & -0.047 \\
\hline$(2 \mp 7 / 2)^{-1}$ & -0.056 & -0.053 & -0.062 & -0.054 \\
\hline$(1 \mathrm{~h} 9 / 2)^{-1}$ & -0.010 & -0.071 & -0.978 & -0.080 \\
\hline
\end{tabular}

Table 4: Proton and charge radius variation for the addition of valence particles and holes. 
variation in $\delta\left\langle r^{2}\right\rangle$ is in fact very similar for both of these Skyrme interactions. Our further discussion is based on results obtained with the skyrme III interaction.

Table 4 compares also the radius variation for the (point) proton distribution with that for the charge which includes in addition the neutron finite size and relativistic spin-orbit corrections (Bertozzi et al. 1972). The spin-orbit correction is the most important in this case. Finally we can compare the calculated values of $\delta\left\langle r^{2}\right\rangle_{c h}$ in ${ }^{209} \mathrm{~Pb}$ (in a $2 \mathrm{~g} 9 / 2$ single-particle state) and ${ }^{207} \mathrm{~Pb}$ (in a $3 \mathrm{p} 1 / 2$ single-particle state) to experiment (see Table 3). For these two states the orbital dependence is small. The values of $\delta\left\langle r^{2}\right\rangle_{c h}$ are about $20 \%$ larger than in the droplet model, and the agreement with experiments appears to be improved.

Next we consider the first-order calculation for even-even $\mathrm{Pb}$ isotopes. For the one-body monopole operator, $\delta\left\langle r^{2}\right\rangle_{\mathrm{Ch}}$ in a manyparticle wavefunction is given by a sum of the one-particle shifts over the occupation probabilities for a particle or hole in those states:

$$
\delta\left\langle r^{2}\right\rangle_{c h}=\sum_{n l j} \quad O_{n l j} \quad \delta\left\langle r^{2}\right\rangle_{c h}^{n l j}
$$

where $o_{n l j}$ is the occupation number $((2 j+1)$ times the occupation probability).

The occupation numbers for the ground states of some $\mathrm{Pb}$ isotopes have been obtained in shell-model calculations by (McGrory and Brown 1982, and Borchert et a1. 1982) using the wavefunctions of McGrory and Kuo (1975). We have calculated the occupation numbers for all of even-even $\mathrm{Pb}$ isotopes with a pairing interaction $(G=0.15)$ using the BCS method. The single-particle energies used and the resulting occupation numbers are given in Table 5. The shell-model and BCS occupation numbers are in fact very similar and $\delta\left\langle r^{2}\right\rangle_{c h}$ depends very little on which one are used. The difference between the results given in Table 3 and the results obtained by McGrory and Speth (McGrory and Brown 1982, and Borchert et al. 1982) is due to the difference in the $1 \mathrm{~h} 9 / 2$ single-particle shift mentioned above. We have 
also carried out more exact calculations by repeating the Hartree-Fock calculation for each nucleus with constraints on the occupation numbers. As an example, this gave a $\delta\left\langle r^{2}\right\rangle_{\mathrm{Ch}}$ value for ${ }^{200} \mathrm{~Pb}$ of $-0.509 \mathrm{fm}^{2}$ compared to the $-0.489 \mathrm{fm}^{2}$ obtained with Eq. 5.1. The reason for this difference is that in the HF calculation the neutron orbits themselves change a little as neutrons are added. This difference is rather small and systematic, but in order to preserve the simplicity involved in using Eq. 5.1 only these results are given in Table 3.

$$
\mathrm{nlj} \quad \begin{gathered}
\varepsilon_{\mathrm{nlj}} \\
{[\mathrm{MeV}]}
\end{gathered}
$$

\begin{tabular}{|c|c|c|c|c|c|c|}
\hline $3 d 3 / 2$ & -1.40 & 0.04 & 0.09 & & & \\
\hline $2 g 7 / 2$ & -1.45 & 0.08 & 0.18 & & & \\
\hline $4 \mathrm{~s} 1 / 2$ & -1.91 & 0.03 & 0.06 & & & \\
\hline $3 d 5 / 2$ & -2.37 & 0.11 & 0.24 & & & \\
\hline $1 j 15 / 2$ & -2.52 & 0.34 & 0.71 & & & \\
\hline 1 i $11 / 2$ & -3.16 & 0.45 & 0.92 & & & \\
\hline \multirow[t]{2}{*}{$2 g 9 / 2$} & -3.94 & 0.95 & 1.80 & & & \\
\hline & & $O\left({ }^{206} \mathrm{~Pb}\right)$ & $\mathrm{O}\left({ }^{204} \mathrm{~Pb}\right)$ & $O\left({ }^{202} \mathrm{~Pb}\right)$ & $O\left({ }^{200} \mathrm{~Pb}\right)$ & $\mathrm{O}\left({ }^{198} \mathrm{~Pb}\right)$ \\
\hline$(3 p 1 / 2)^{-1}$ & $-7 \cdot 37$ & 0.72 & 1.08 & 1.27 & 1.40 & 1.50 \\
\hline$(2 £ 5 / 2)^{-1}$ & $-7 \cdot 9$ & 0.60 & 1.33 & 2.06 & 2.71 & 3.26 \\
\hline$(3 \mathrm{p} 3 / 2)^{-1}$ & -8.27 & 0.22 & 0.53 & 0.88 & 1.25 & 1.61 \\
\hline$(1 i 13 / 2)^{-1}$ & -9.00 & 0.31 & 0.72 & 1.23 & 1.83 & 2.53 \\
\hline$(2 £ 7 / 2)^{-1}$ & -9.71 & 0.09 & 0.21 & 0.35 & 0.51 & 0.71 \\
\hline$(1 \mathrm{~h} 9 / 2)^{-1}$ & -10.78 & 0.06 & 0.13 & 0.21 & 0.30 & 0.39 \\
\hline
\end{tabular}

Table 5: Single-particle energies $\left(\varepsilon_{n l j}\right)$ and occupation number (0) as calculated by the BCS method

In comparison with experiment the theoretical fine structure and shell effects obtained are too small, but the existing structure is in the right direction. In particular, the theoretical odd-even staggering between ${ }^{206} \mathrm{~Pb}$ and $210 \mathrm{~Pb}$ is to small compared to experiment. This is due to the small orbital dependence of the single particle shifts. We have not calculated the occupation 
numbers for the other odd isotopes (A<206), but for the same reason we would not expect much theoretical odd-even staggering. In Table 3 just interpolated values for these nuclei are given.

We briefly consider higher order corrections due to possible static or dynamic deformation of $\mathrm{Pb}$ nuclei when going away from the closed neutron shell. In the framework of a generalized collective model such contributions

$$
\delta\left\langle r^{2}\right\rangle_{\operatorname{def}}=\frac{5}{4}\left\langle r^{2}\right\rangle_{\mathrm{O}} \delta\left\langle\beta^{2}\right\rangle
$$

can be related via a sum rule to the electromagnetic transition probabilities and the ground state quadrupole moment $Q$ by

$$
\begin{gathered}
\left\langle B^{2}\right\rangle=\left[\frac{4 \pi}{5 \mathrm{Ze}}\left\langle r^{2}\right\rangle_{0}\right]^{-2}\left[\frac{5}{16 \pi} \frac{\left(2 J_{i}+1\right)\left(2 J_{i}+3\right)}{J_{1}\left(2 J_{i}-1\right)} Q\left(J_{i}\right)^{2}+\right. \\
\left.\sum_{f}^{\sum B\left(E 2 ; J_{i} \rightarrow J_{f}\right)}\right]
\end{gathered}
$$

Here $\left\langle r^{2}\right\rangle_{0}$ is the zeroth order mean square charge radius, and a homogenous charge distribution with only quadrupole deformation is assumed. Generally, the contribution from $Q^{2}$ can be neglected relative to the contribution from the $B(E 2)$ values. The relationship of estimates on this basis to second order perturbation theory with coupling to the quadrupole and other multipole core vibrations is presently being studied (Brown and McGrory 1982).

Unfortunately the experimental information on the $E 2$ transitions in the $\mathrm{Pb}$-isotopes is incomplete. Thus we have supplemented what exists by the shell-model calculations of McGrory (McGrory and Brown 1982 and McGrory 1982). Some results are summarized in Table 6. The shell-model $B(E 2)$ values calculated with Woods-Saxon wavefunctions have been normalized to the ${ }^{206} \mathrm{~Pb}$ experimental value by using an $\mathrm{E} 2$ neutron effective charge of $1.1 \mathrm{e}$ (the neutron effective charge itself arises from a coupling in first order between the particle and quadrupole states of the ${ }^{208} \mathrm{~Pb}$ core). 


\begin{tabular}{|c|c|c|c|c|}
\hline Nucleus & $\begin{array}{c}\mathrm{B}\left(\mathrm{E} 2 ; 0^{+}-2{ }^{+}\right) \\
{\left[\mathrm{e}^{2} \mathrm{fm}^{4}\right]}\end{array}$ & & $\begin{array}{l}B(E 2 ; g-f) \\
{\left[e^{2} \mathrm{fm}^{4}\right]}\end{array}$ & $\begin{array}{c}\delta\left\langle r^{2}\right\rangle \\
{\left[f m^{2}\right] .}\end{array}$ \\
\hline & Exp. & Theor. (a) & Theor. (a) & Theor. \\
\hline $210_{\mathrm{Pb}}$ & $520^{(b)}$ & 533 & $(600)^{(c)}$ & 0.007 \\
\hline $207 \mathrm{pb}$ & & & 353 & 0.004 \\
\hline $206^{2} \mathrm{~Pb}$ & $920(65)^{(d)}$ & 920 & 1093 & 0.013 \\
\hline${ }^{205} \mathrm{~Pb}$ & & & 1127 & 0.014 \\
\hline${ }^{204} \mathrm{~Pb}$ & $1675(25)^{(d)}$ & 1537 & 1864 & 0.023 \\
\hline $202 \mathrm{~Pb}$ & - & 1964 & 2375 & 0.029 \\
\hline${ }^{200} \mathrm{~Pb}$ & - & 2326 & 2864 & 0.036 \\
\hline
\end{tabular}

(a) McGrory and Brown (1982) and McGrory, private communication

(b) Ellegard et al. (1971);

(c) Estimate based on the calculations for the other even-even nuclei;

(d) Lederer and Shirley (1979)

Table $6: B(E 2)$ values and their contributions to $\delta\left\langle r^{2}\right\rangle_{\mathrm{ch}}$

With this effective charge the accurately measured $B(E 2)$ value for ${ }^{204} \mathrm{~Pb}$ as well as an experimental estimate for the $0^{+} \rightarrow 2_{1}^{+}$in $210 \mathrm{~Pb}$ based on an analysis of triton inelastic scattering data (Ellegaard et al. 1971) are in agreement with theory.

The shell-model calculations is then used to obtain the summed $B(E 2)$ values to all excited $2^{+}$states as well as the sum for a few odd-even nuclei for which the calculations were feasible. The contributions $\left(\delta\left\langle r^{2}\right\rangle_{\text {def }}\right.$ ) to $\delta\left\langle r^{2}\right\rangle_{c h}$ (see Table 6 ) are combined with the above first order calculations to obtain the total variation in charge radii as given in Table 3 (see columns with $+\mathrm{E} 2$ ).

These second-order corrections contain more structure in terms of odd-even staggering. The essential reason for this can be understood in the $j^{\text {n }}$ shell model (Sorensen 1966). The agreement with experiment is now better for the even isotopes but worse 
for the odd-even isotopes. Noting the second-order corrections are always positive, the only way at present to make the theory consistent would be to increase the first-order shift for ${ }^{207} \mathrm{~Pb}$ by $20 \%$ to. $0.076\left[\mathrm{fm}^{2}\right]$ (see the column with (Emp.Sh) in Table 3). Then there is the possibility that the remaining deviation for the lighter $\mathrm{Pb}$ isotopes could be improved by a positive contribution from other second-order corrections resulting from the higher multipoles such as $\mathrm{E} 4$.

\section{CONCLUDING REMARKS}

The high resolution measurements of the isotope shifts and hyperfine structure in a fairly long series of stable and radioactive $\mathrm{Pb}$ isotopes point out interesting nuclear features.

(i) Before the closure of the $\mathrm{N}=126$ neutron shell the overall variation of $\delta\left\langle r^{2}\right\rangle_{C h}^{A}-208$ agrees rather well with predictions of the spherical nuclear droplet model.

(ii) There is a significant odd-even staggering in the variation of the charge radii, which is not reproduced by theoretical approaches.

(iii) The closure of the $N=126$ neutron shell affects distinctly the $\delta\left\langle r^{2}\right\rangle_{c h}$ variation: $\left\langle r^{2}\right\rangle_{c h}$ increases more rapidly when the neutrons start filling a new shell than when the shell is nearly closed.

(iv) The value obtained for the magnetic moment of ${ }^{209} \mathrm{~Pb}$ agrees with the value deduced indirectly for a $\mathrm{g} 9 / 2$ neutron form the $7^{-}$state in $210_{\mathrm{Bi}}$ (Baba et al. 1972), again confirming the absence of any anomaly in the orbital and spin $g$ factors (Arima and Huang-lin 1972).

(v) The ground state spin of ${ }^{199} \mathrm{~Pb}$ has been determined to be $I=3 / 2$ removing an erroneous result quoted in the literature.

The shell model calculations performed are generally in good agreement with experiment, but they have difficulties in reproducing the odd-even staggering and the jump in $\delta\left\langle r^{2}\right\rangle_{C h}$ 
after closing the shell. Higher order corrections (deformation effects) have been found to be important for improving the results of theoretical studies based on microscopic nuclear structure approaches.

It is pleasure to acknowledge stimulating discussions with Profs. P. Brix, E. Matthias and O.W.B. Schult. We would like to thank Dr. M. Zirnbauer (Theoretical Physics Department Oxford) whose BCS program was used, and Dr. D. Frölich and A. Steiger for their help during the measurements. 


\section{REFERENCES}

Arima A. and Huang-Lin I J 1972 Phys. Lett. 41B 435

Baba C V K, Faestermann T, Fossan D B and Proetel D 1972 Phys.Rev. Lett. $2 \underline{9} 496$

Beiner M, Flocard H, Van Gai N and Quentin P 1975 Nucl. Phys. A 23829

Bertozzi W, Friar J, Heisenberg J and Negele J 1972 Phys.Lett. $41 \mathrm{~B} \quad 408$

Borchert G I, Schult O W B, Speth J, Hansen P G, Johnson B, Ravn H L and McGrory J B 1982 (submitted to Nuovo Cimento)

Boyd G D and Kleinman D A 1968 J. Appl. Phys. 39347

Brix P, V.Buttlar H, Houtermanns F C and Kopfermann H 1952

Z. Physik 133192

Brown B A and McGrory J B 1982 (unpublished results)

Ellegaard C, Barnes P D, Flynn E R and Igo G J 1971

Nucl. Phys. A 1621

Fricke G, Miska $H$ and Rychel D 1981 (private communication)

Fuller G H 1976 J. Phys. Chem. Ref. Data $\underline{5} 835$

Hüffer W 1982 thesis, Universität Köln, (private communication)

Kesseler D, Mes H, Thompson A C, Anderson H L, Dixit M S, Hargrove C K and McKee R J 1975 Phys. Rev. C11 1719

Kopfermann H 1932 Z Physik $\underline{75} 363$

Kuhn H G 1969 "Atomic Spectra" (Second edn, Longmans, Grun and Co Ltd, London)

Lederer C M and Shirley V S 1979 Table of Isotopes (John Wiley and Sons New York)

Lindgren I, Timmermann A and Matthias E 1981 European Conference Abstracts 5A 235 (ECAP Heidelberg)

McGrory J B 1982 (private communication) 
McGrory J B and Kuo T T S 1975 Nucl. Phys. A 247283

McGrory J B and Brown B A 1982 Conference on Lasers in Nuclear Physics Oak Ridge Tenn (April 21-23)

Myers W D 1977 Droplet Model of the Nucleus (IFI/Plenum Data Co New York)

Myers W D 1982 Conference on Lasers in Nuclear Physics Oak Ridge Tenn (April 21-23)

Moscatelli F A $1980 \mathrm{Ph}$ D thesis New York University (unpub1)

Moscatelli F A, Redi O, Wiggins R L and Stroke H H 1981

European Conference Abstracts $\underline{5}$ A 225 (ECAP Heidelberg)

Nowicki G, Bekk K, Göring S, Hanser A, Rebel H and Schatz G 1978 Phys. Rev. C18 2369

Richel H, Albouy G, Auger G, Hanappe F, Lagrange J M, Pautrat M, Roulet C, Sergolle H and Vanhorenbeck J 1978 Nucl. Phys. A303 483

Seltzer E C 1969 Phys. Rev. 1881916

Sorensen R A 1966 Phys. Lett. $\underline{21} 333$

Speth J, Zamick I and Ring P 1974 Nucl. Phys. A 2321

Speth J 1981 (private communication)

Thompson R C, Hanser A, Bekk K, Meisel G and Frölich D 1981

Z. Phys. A 30589 
APPENDIX

Positions of the hyperfine splitting components relative to the ${ }^{208} \mathrm{~Pb}$ resonance line

\begin{tabular}{|c|c|c|c|}
\hline Isotop & I & $\mathbf{F}$ & Position $[\mathrm{MHz}]$ \\
\hline \multirow[t]{3}{*}{199} & $3 / 2$ & $5 / 2$ & $-17733(15)$ \\
\hline & & $3 / 2$ & $-4417(15)$ \\
\hline & & $1 / 2$ & $3545 \quad(15)$ \\
\hline \multirow[t]{3}{*}{201} & $5 / 2$ & $3 / 2$ & $-14753.1(8)$ \\
\hline & & $5 / 2$ & $-9736.1(5)$ \\
\hline & & $7 / 2$ & $-2708.5(4)$ \\
\hline \multirow[t]{3}{*}{$202 \mathrm{~m}$} & 9 & 10 & $-7938.3(8)$ \\
\hline & & 9 & $-6003.6(6)$ \\
\hline & & 8 & $-4374.2(6)$ \\
\hline \multirow[t]{3}{*}{203} & $5 / 2$ & $3 / 2$ & $-12897.8(7)$ \\
\hline & & $5 / 2$ & $-7780.3(6)$ \\
\hline & & $7 / 2$ & $-651.1(4)$ \\
\hline \multirow[t]{3}{*}{205} & $5 / 2$ & $3 / 2$ & $-11135.8(5)$ \\
\hline & & $5 / 2$ & $-5807.6(3.5)$ \\
\hline & & $7 / 2$ & $1570.2(2)$ \\
\hline \multirow[t]{2}{*}{207} & $1 / 2$ & $1 / 2$ & $-10198 \cdot 1(3)$ \\
\hline & & $3 / 2$ & $3012.7(3)$ \\
\hline \multirow[t]{3}{*}{209} & $9 / 2$ & $11 / 2$ & $-9175(20)$ \\
\hline & & $9 / 2$ & $4180 \quad(15)$ \\
\hline & & $7 / 2$ & $15165(15)$ \\
\hline
\end{tabular}

